\title{
Anticodon $\mathrm{G}$ recognition by tRNA synthetases mimics the tRNA core
}

\author{
Liron Klipcan ${ }^{1}$, Mark Safro ${ }^{2}$, and Paul Schimmel ${ }^{1,}{ }^{*}$ \\ ${ }^{1}$ Departments of Molecular Biology and Chemistry and The Skaggs Institute for Chemical Biology, \\ The Scripps Research Institute, 10550 N. Torrey Pines Road, La Jolla, CA 92037, USA \\ ${ }^{2}$ Department of Structural Biology, Weizmann Institute of Science, 76100 Rehovot, Israel
}

\section{Abstract}

Ancient mechanisms for nucleotide base recognition in the RNA world were candidates for mimicking by early proteins like tRNA synthetases. In the core of the tRNA, conserved G22 interacts with 2 internal bases in a complex further stabilized by stacking interactions. This particular tRNA format for $\mathrm{G}$ recognition is shown here to be adapted by 9 different and even nonhomologous anticodon binding domains (ABDs) of tRNA synthetases, where amino acid side chains mimic all of the tRNA G22 base interactions. We offer the possibility that mimicking this RNA-based mechanism for guanine recognition was perhaps one of the selective pressures for choosing amino acids for the genetic code.

\section{Guanine recognition in the tRNA core}

In the hypothesized RNA world before the appearance of proteins, highly evolved RNA molecules carried out binding and catalytic events. Later, in the transition from the RNA world to the theatre of proteins, the emerging family of aminoacyl tRNA synthetases (aaRS) established the genetic code by catalyzing attachment of amino acids to their cognate tRNAs that harbored the anticodon triplet specific to the attached amino acid (Box 1)[1]. This transition would be facilitated if early proteins could substitute for catalytic and ligandbinding RNAs by using amino acid side chains to mimic the interactions in the 'RNA world'. Because RNA-based guanine recognition plays a prominent role in the 'RNA world' of splicing and hydrolytic cleavage, G-recognition was analyzed in detail[2]. Possibly, the mechanism for protein-based recognition of $\mathrm{G}$ in the tRNA anticodon was patterned after the tRNA-based systems and played a role in amino acid selections for the modern genetic code.

In the core of the tRNA 3D structure is a complex series of H-bonds and aromatic/ hydrophobic interactions that includes the semi-invariant C13-G22-G46 base triple, where G22 is embraced by H-bonding to G46 and C13 along its Hoogsteen and Watson-Crick edge, respectively, and by a hydrophobic stacking interaction with A14 (Figure 1a,b) [3, 4]. Amongst the available co-crystal synthetase-tRNA structures, most or all of these interactions were strikingly found to be closely mimicked by interactions of the anticodon binding domains (ABDs) of tRNA synthetases that use $\mathrm{G}$ as an important tRNA identity element (see below) [5].

\footnotetext{
(C) 2012 Elsevier Ltd. All rights reserved.

*To whom correspondence should be addressed. schimmel@ scripps.edu.
}

Publisher's Disclaimer: This is a PDF file of an unedited manuscript that has been accepted for publication. As a service to our customers we are providing this early version of the manuscript. The manuscript will undergo copyediting, typesetting, and review of the resulting proof before it is published in its final citable form. Please note that during the production process errors may be discovered which could affect the content, and all legal disclaimers that apply to the journal pertain. 


\section{Recognition of $\mathrm{G}$ in anticodon triplet by aaRSs}

Five different structures of aaRSs that target anticodons that use G34 as an identity element were compared [6-13] (Figure 2a). Because this set of 5 aaRSs (class II PheRS, SepRS, AspRS and class I CysRS, TyrRSs) belong to different classes and subclasses, with widely diverging architectures, G34 itself was used as the point on which to superimpose the 5 complexes of anticodons bound to their respective ABDs. While none of the $5 \mathrm{ABD}$ structures have sequence or structural similarity (Figure 2b), in all cases the specific recognition of G34 is achieved by a similar mechanism involving three amino acids (Figure $2 \mathrm{c}$ and Figure 3). First is the bidentate "Watson-Crick" interaction of the carboxylic group of Asp (Asp364-PheRS, Asp436-CysRS and Asp286-TyrRS) or Glu (Glu188-AspRS, Glu420SepRS) with N1 and N2 of G34. Second is the "Hoogsteen" hydrogen bond between N7 (and in some cases O6) of G34 and positively charged Arg, Lys or His (Arg414-PheRS, Arg427-CysRS, Arg492-SepRS His283-TyrRS, Lys142-AspRS) (Figure 2c and Figure 3). Interestingly, before the first structure of a nucleic acid-protein was solved Rich and coworkers predicted that, based on the A14-C13-G22-G46 base triple, protein-directed recognition of $\mathrm{G}$ would involve a specific interaction between Arg (Lys, His) and N7 [14]. Third is the recruitment of an aromatic amino acid side chain for "face-to-face" (Phe366PheRS, Trp432-CysRS, Phe261-TyrRS) or "edge-to-face" (Phe526-SepRS, Phe127-AspRS) interactions. Thus, in spite of the wide divergence of structures, the same format for recognition of $\mathrm{G}$ has been imbedded through a process of convergent evolution. This format for selecting $\mathrm{G}$ must, therefore, be robust.

If this observation is true, then virtual docking of a tRNA onto its synthetase, when the structure of the complex is not available, should reveal the same pattern for recognition of G34 (Figure 4). For this purpose, the structure of AsnRS (which is closely related to AspRS) was superimposed onto the AspRS/tRNA ${ }^{\text {Asp }}$ complex [15]. In this model, the recognition of G34 is achieved by the bidentate interaction of Glu89 with N1 and N2 of G34 (Glu188 in AspRS), the interaction of N7 with Lys34, and the "edge-toface" interaction of G34 with Phe36 (Phe127 in AspRS). While Glu89 of AsnRS is orthologous to Glu188 of AspRS, importantly, Lys34 of AsnRS is not orthologous (in the sequence) to its counterpart in AspRS (Figure 4). In a different vein, and as another example of the predictive value of using the principles of anticodon G-recognition described here, a disease-causing Grecognition mutation in human mitochondrial tRNA ${ }^{\text {Phe }}$ has been compensated by a rational mutation in the synthetase (Box 2).

In considering the other positions in the anticodon, ProRS (NGG anticodon) and ThrRS (NGU anticodon) both use $\mathrm{G}$ as an anticodon identity determinant and have available structures (Figure 2a) [16, 17]. Although GlnRS interacts with the G36 anticodon nucleotide, that interaction is not critical for tRNA identity, therefore we did not include it in the comparison. Phylogenetic analysis suggests that ProRS and ThrRS are closely related and form a monophyletic clade [18]. Indeed, the mechanisms of recognition of G35 by ProRS and ThrRS are almost identical and have the same pattern as seen with the recognition of G36 by ProRS: there is a bidentate interaction of N1 and N2 with a carboxyl side chain and a bonding between N7 and a positively charged Arg or Lys (Figure 3). For G35, the additional hydrophobic aromatic interaction seen with G36 is replaced by a hydrophobic interaction with isoleucine (Ile295 in ProRS and Ile547 ThrRS).

\section{Concluding remarks}

The widely divergent structures of the ABDs of tRNA synthetases locally converged to embrace $\mathrm{G}$ identity elements in tRNA anticodons, with semi-conserved interactions that closely mimic how G22 fits into the core of the universal tRNA structure. Possibly, this 
ancient RNA mechanism for guanine recognition played a key role in going from the RNA world to the theatre of proteins. For example, mimicking the RNA-based format for G recognition could have been one of the selective pressures for choosing pre-existing amino acids like Arg, Lys, His, Glu, Asp, Phe for the universal genetic code. In addition, the robust nature of G-recognition by these amino acids could have influenced or biased early pathways for amino acid creation. Finally, while the RNA world hypothesis as an explanation for the origin of the genetic code and of life is unproven, the results here are easily compatible with the hypothesis.

\section{Acknowledgments}

We thank Professors Gerald Joyce, Jamie Williamson, and Xiang-Lei Yang for comments and advice. This work was supported by grant GM15539 and 23562 from the NIH, by a fellowship from the National Foundation for Cancer Research, by Israel Science Foundation (grant 710262 to M.S.), and by the Kimmelman Center for Biomolecular Structure and Assembly at Weizmann Institute of Science (M.S.).

\section{References}

1. Schimmel P. Development of tRNA synthetases and connection to genetic code and disease. Protein Sci. 2008; 17:1643-1652. [PubMed: 18765819]

2. Lilley DMJ. Mechanisms of RNA catalysis. Philosophical Transactions of the Royal Society B: Biological Sciences. 2011; 366:2910-2917.

3. Hoogsteen K. The crystal and molecular structure of a hydrogen-bonded complex between 1methylthymine and 9-methyladenine. Acta Crystallographica. 1963; 16:907-916.

4. Rich A, RajBhandary UL. Transfer RNA: Molecular Structure, Sequence, and Properties. Annual Review of Biochemistry. 1976; 45:805-860.

5. Giege R, et al. Universal rules and idiosyncratic features in tRNA identity. Nucleic Acids Res. 1998; 26:5017-5035. [PubMed: 9801296]

6. Fukunaga R, Yokoyama S. Structural insights into the first step of RNA-dependent cysteine biosynthesis in archaea. Nat Struct Mol Biol. 2007; 14:272-279. [PubMed: 17351629]

7. Hauenstein S, et al. Shape-selective RNA recognition by cysteinyl-tRNA synthetase. Nat Struct Mol Biol. 2004; 11:1134-1141. [PubMed: 15489861]

8. Klipcan L, et al. Crystal structure of human mitochondrial PheRS complexed with tRNA(Phe) in the active "open" state. J Mol Biol. 2012; 415:527-537. [PubMed: 22137894]

9. Kobayashi T, et al. Structural basis for orthogonal tRNA specificities of tyrosyl-tRNA synthetases for genetic code expansion. Nat Struct Biol. 2003; 10:425-432. [PubMed: 12754495]

10. Cavarelli J, et al. Yeast tRNA(Asp) recognition by its cognate class II aminoacyl-tRNA synthetase. Nature. 1993; 362:181-184. [PubMed: 8450889]

11. Yaremchuk A, et al. Class I tyrosyl-tRNA synthetase has a class II mode of cognate tRNA recognition. Embo J. 2002; 21:3829-3840. [PubMed: 12110594]

12. Goldgur Y, et al. The crystal structure of phenylalanyl-tRNA synthetase from thermus thermophilus complexed with cognate tRNAPhe. Structure. 1997; 5:59-68. [PubMed: 9016717]

13. Eiler S, et al. Synthesis of aspartyl-tRNA(Asp) in Escherichia coli--a snapshot of the second step. Embo J. 1999; 18:6532-6541. [PubMed: 10562565]

14. Seeman NC, et al. Sequence-specific recognition of double helical nucleic acids by proteins. Proc Natl Acad Sci U S A. 1976; 73:804-808. [PubMed: 1062791]

15. Iwasaki W, et al. Structural basis of the water-assisted asparagine recognition by asparaginyl-tRNA synthetase. J Mol Biol. 2006; 360:329-342. [PubMed: 16753178]

16. Cusack S, et al. tRNA(Pro) anticodon recognition by Thermus thermophilus prolyl-tRNA synthetase. Structure. 1998; 6:101-108. [PubMed: 9493271]

17. Sankaranarayanan $\mathrm{R}$, et al. The structure of threonyl-tRNA synthetase-tRNA( Thr) complex enlightens its repressor activity and reveals an essential zinc ion in the active site. Cell. 1999; 97:371-381. [PubMed: 10319817] 
18. Ribas de Pouplana L, et al. Structure-based phylogeny of class IIa tRNA synthetases in relation to an unusual biochemistry. J Mol Evol. 2001; 53:261-268. [PubMed: 11675586]

19. Eriani G, et al. Partition of tRNA synthetases into two classes based on mutually exclusive sets of sequence motifs. Nature. 1990; 347:203-206. [PubMed: 2203971]

20. Rodin SN, Ohno S. Four primordial modes of tRNA-synthetase recognition, determined by the (G,C) operational code. Proc Natl Acad Sci U S A. 1997; 94:5183-5188. [PubMed: 9144212]

21. Ribas de Pouplana L, Schimmel P. Aminoacyl-tRNA synthetases: potential markers of genetic code development. Trends Biochem Sci. 2001; 26:591-596. [PubMed: 11590011]

22. Ling J, et al. Pathogenic mechanism of a human mitochondrial tRNAPhe mutation associated with myoclonic epilepsy with ragged red fibers syndrome. Proc Natl Acad Sci U S A. 2007; 104:15299-15304. [PubMed: 17878308] 


\section{Box 1 Two classes of aaRSs}

The aaRSs establish the genetic code by specific aminoacylations of tRNAs with their cognate amino acid. The aaRSs are partitioned into two different classes. Class I synthetases have catalytic domains containing the classical nucleotide-binding Rossmann fold while class II aaRSs are built around an antiparallel $\beta$-sheet flanked by $a$-helices [19]. Interestingly, the anticodon binding domain, which is separate from the catalytic unit, appears to be a later addition to synthetases and, unlike the two types of catalytic domains (designated as class I and II), occurs in more than 7 different and unrelated architectural formats. The subdivision of the aaRSs into two classes (with roughly equal members in each), and other considerations, led to the hypothesis that distinctions between classes evolves from a gene whose sense and antisense strands code for ancestors of two ancient aaRS classes [20,21].

Box 1. Figure I. The two subclasses of aminoacyl-tRNA synthetases. The different subclasses that form each class are shown in different colors.

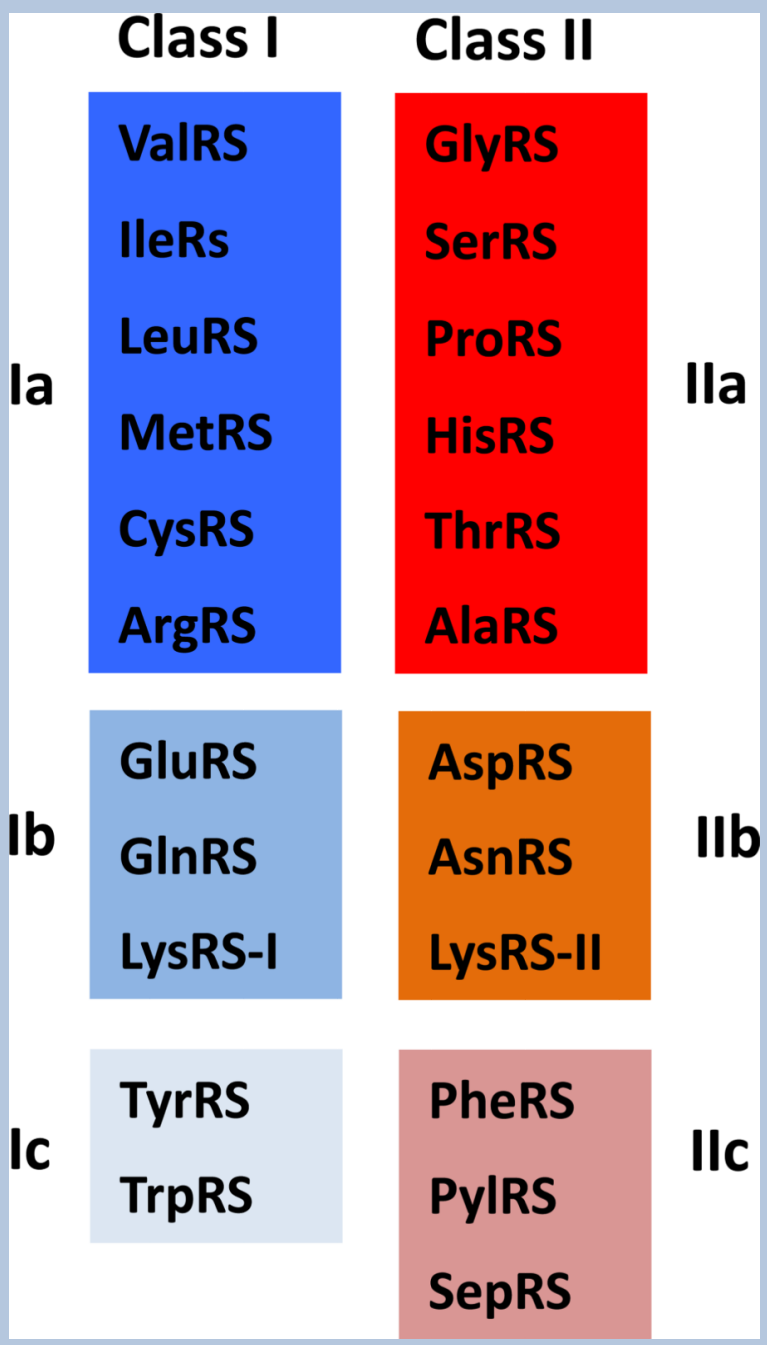




\section{Box 2 Treatment of a human mitochondrial disease caused by a tRNA anticodon mutation}

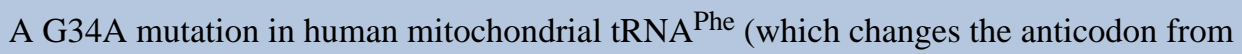
GAA to AAA) is associated with myoclonic epilepsy with ragged red fibers (MERFF). This mutation results in a deficient aminoacylation of mitochondrial PheRS. In an attempt to rescue the aminoacylation defect of G34A tRNA ${ }^{\text {Phe }}$, human mitochondrial PheRS was rationally modified by an Asp364Asn substitution in order to restore hydrogen bonding with A34 [22] . The resulting Asp364Asn PheRS showed significantly improved aminoacylation efficiency and thus suggested, in principle, a potential route to treat the MERRF syndrome.

Box 2 Figure I. (a) Recognition of G34 by human mitochondrial PheRS (PDB ID 3TUP). (b) Model for G34A mitochondrial tRNA ${ }^{\text {Phe }}$ recognition. (c) Proposed recognition of G34A tRNA ${ }^{\text {Phe }}$ by Asp364Asn PheRS.

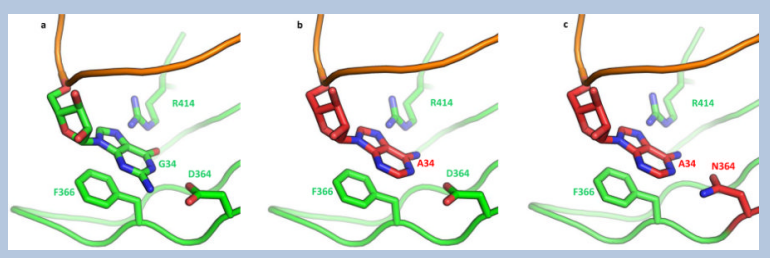


a

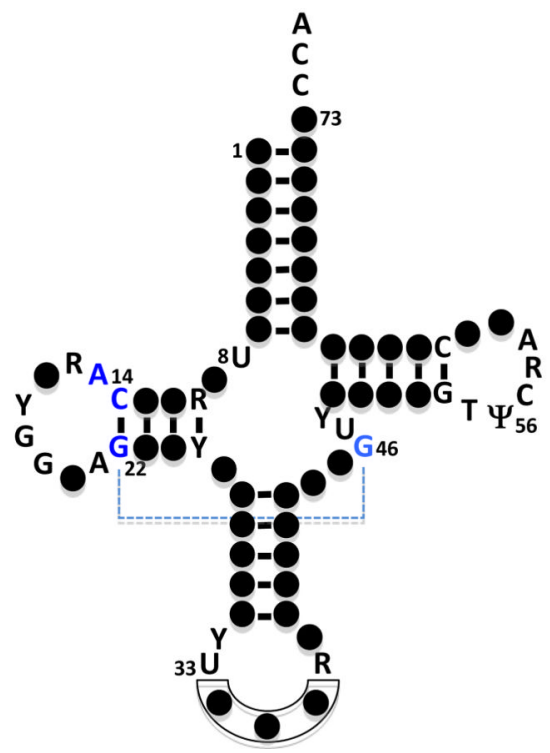

b

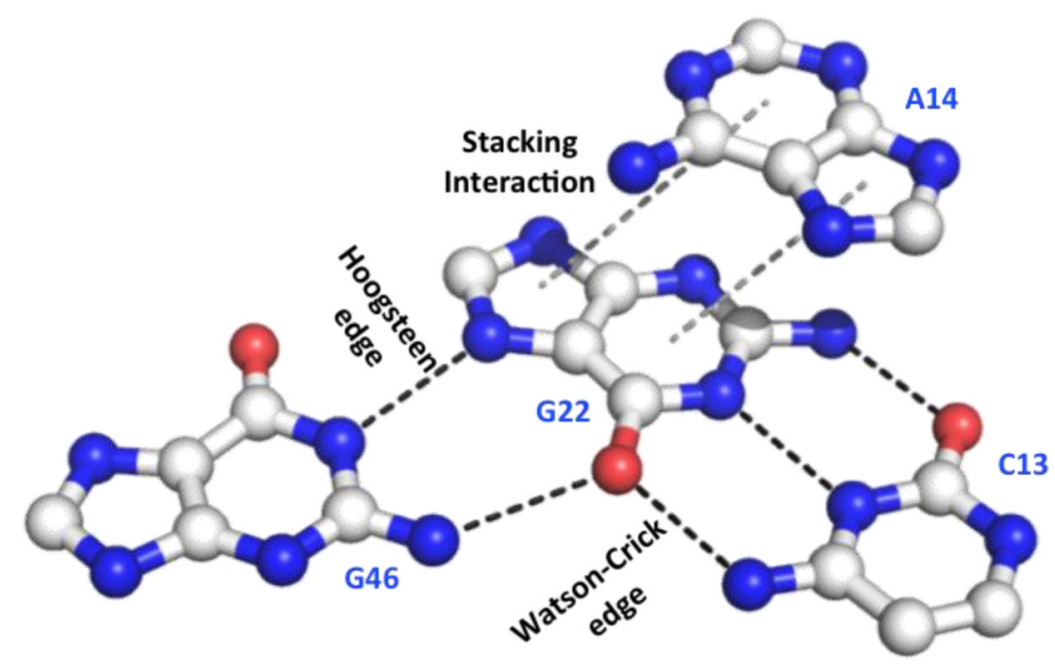

Figure 1. Common recognition of guanine by RNA and proteins

(a) A cloverleaf diagram of tRNA, with the invariant residues annotated with letters corresponding to specific bases and the A14-C13-G22-G46 interaction shown in blue and indicated by a line. The anticodon is enclosed in a box. $\mathrm{R}=$ purine, $\mathrm{Y}=$ pyrimidine, and psi=pseudouridine. (b) Recognition of guanine in the A14-C13-G22-G46 tertiary structure of yeast tRNA ${ }^{\text {Phe is }}$ shown in stick-ball representation. The dashed lines connect atoms engaged in $\mathrm{H}$-bonding interactions. 


$\begin{array}{lll}\text { TyrRS } & \text { G34 } & \checkmark \\ \text { PheRS } & \text { G34 } & \checkmark \\ \text { AspRS } & \text { G34 } & \checkmark \\ \text { AsnRS } & \text { G34 } & { }^{*} \\ \text { SepRS } & \text { G34 } & \checkmark \\ \text { CysRS } & \text { G34 } & \checkmark \\ \text { HisRS } & \text { G34 } & - \\ \text { ProRS } & \text { G35 G36 } & \checkmark \\ \text { ThrRS } & \text { G36 } & \checkmark \\ \text { GInRS } & \text { G36 } & x\end{array}$

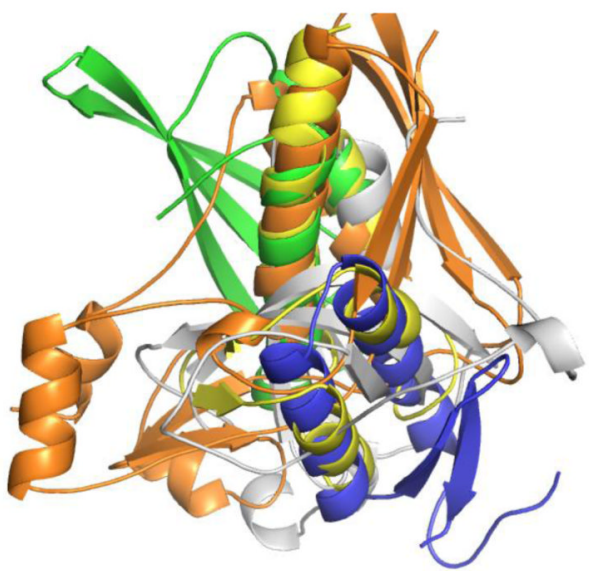

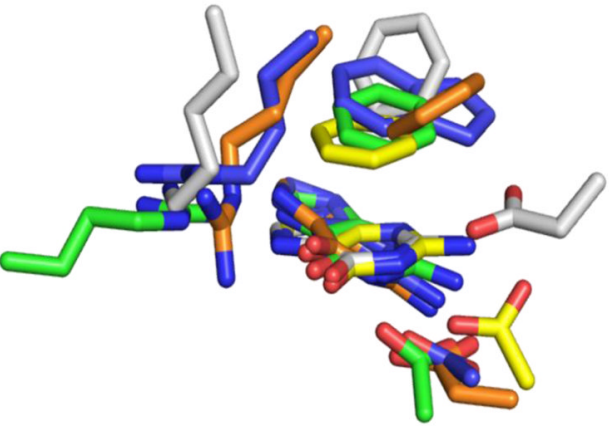

Figure 2. Involvement of anticodon $G$ in tRNA identity

(a) A listing of tRNAs with a $\mathrm{G}$ in their anticodons. Those marked with a check have a common mechanism for $\mathrm{G}$ recognition based on published structures of synthetase-tRNA complexes; * indicates a common mechanism based on structural modeling; - indicates that a complex structure is unavailable; $\times$ indicates a different mechanism for recognition of $G$ in a case where $\mathrm{G}$ is not a major identity element. (b) Superposition of anticodon binding domains of Methanococcus jannaschii TyrRS (PDB ID 1J1U) (yellow), Archeoglobus fulgidus SepRS (PDB ID 2DU3) (orange), Homo sapiens mitochondrial PheRS (PDB ID 3TUP) (green), Escherichia coli CysRS (PDB ID 1U0B) (blue), Saccharomyces cerevisiae AspRS (grey) (PDB ID 1ASY). (c) Superposition of TyrRS (yellow), SepRS (orange), PheRS (green), CysRS (blue), AspRS (grey) around G34. 
TyrRS
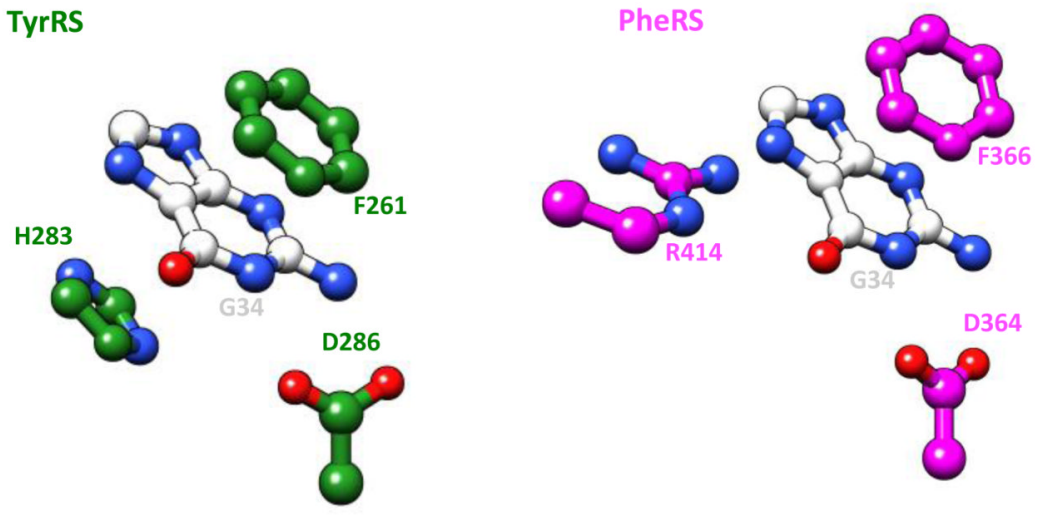

CysRS

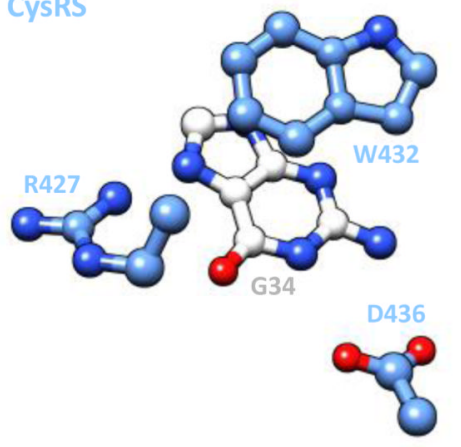

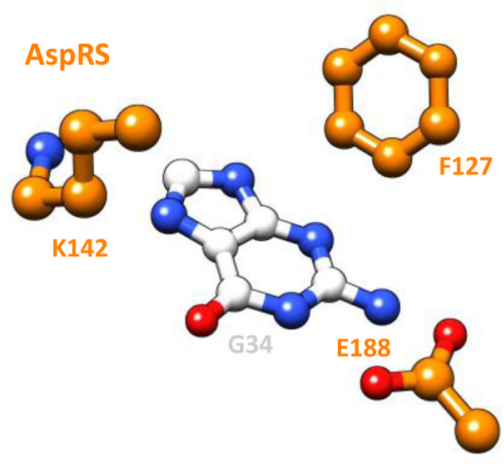
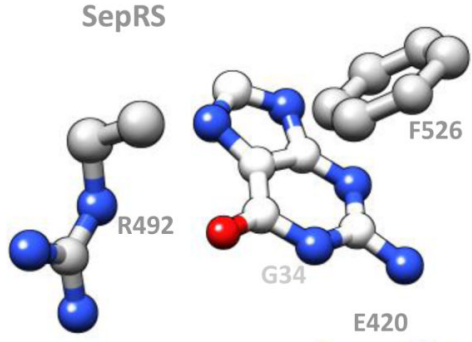

E420
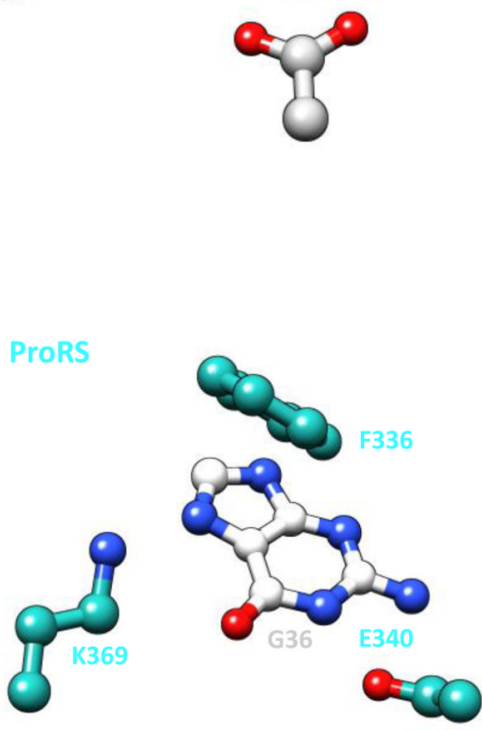

Figure 3.

A common mechanism for recognition of guanine. Recognition of G34 by TyrRS (green), SepRS (grey), PheRS (pink), CysRS (blue), AspRS (orange ) and of G36 by Thermus thermophilus ProRS (PDB ID 1H4S) (light blue). The colors are used to highlight individually the different synthetases. 


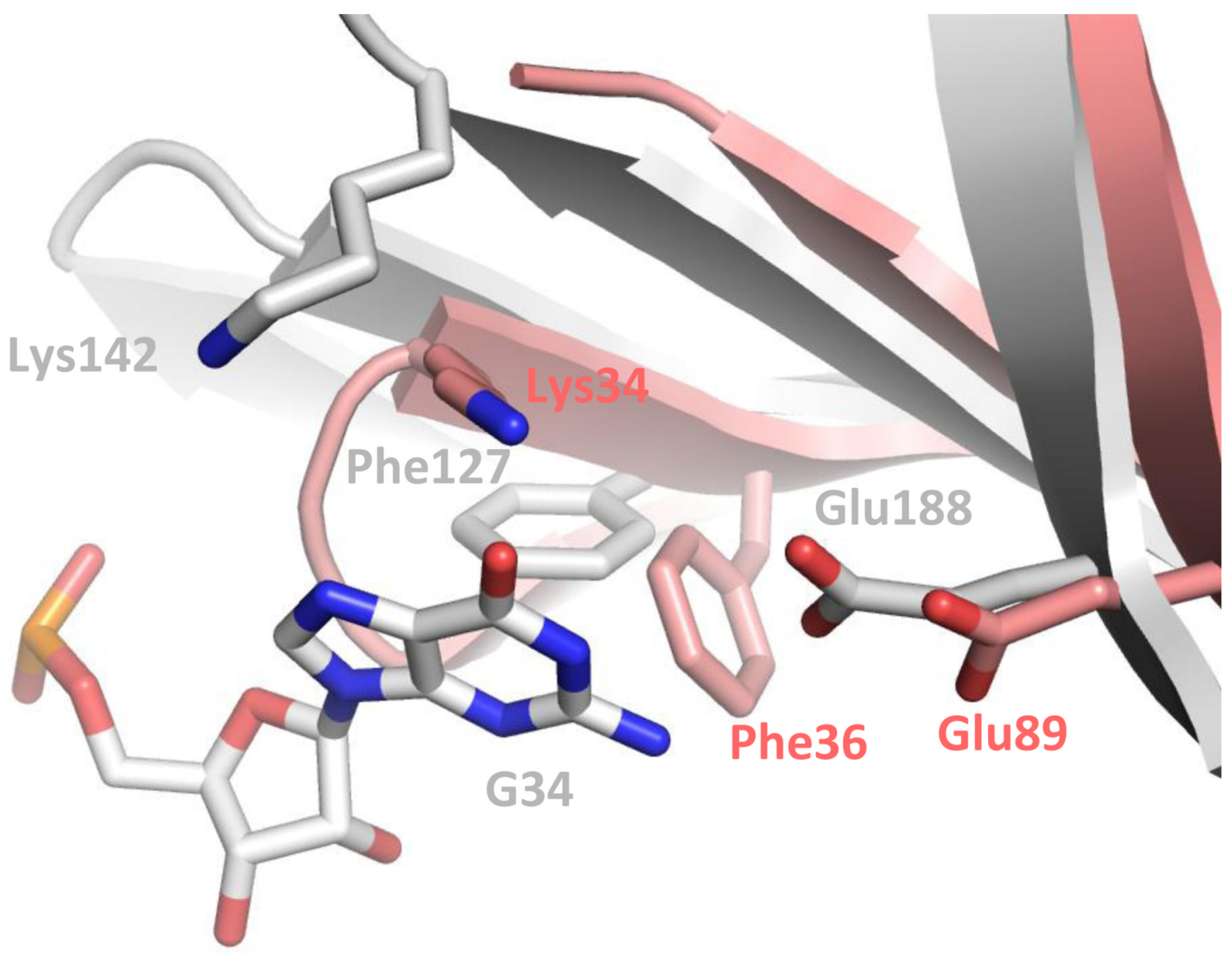

Figure 4.

Modeling of G34 recognition by Pyrococcus horikoshii AsnRS (PDB ID 1X55) (pink), and AspRS (grey) 